\title{
Pesticides in tap water may increase food allergy risk
}

Published online: 07 December 2012

(C) Springer Healthcare 2012

medwireNews: Having a high level of urinary dichlorophenols is associated with the presence of food sensitization and allergies, say researchers.

"Our research shows that high levels of dichlorophenol-containing pesticides can possibly weaken food tolerance in some people, causing food allergy," said lead study author Elina Jerschow (Albert Einstein College of Medicine, Bronx, New York, USA) in a press statement.

"This chemical is commonly found in pesticides used by farmers and consumer insect and weed control products, as well as tap water," she added.

The authors of the study, published in the Annals of Allergy, Asthma, and Immunology, analyzed data collected from 2211 people aged 6 years or older who participated in the National Health and Nutrition Examination Survey 2005-2006. Of these, 411 had at least one food allergy and 1016 at least one environmental allergy.

High exposure to dichlorophenols was defined as having urinary levels at the $75^{\text {th }}$ percentile or above. People with a high level of exposure were a significant $80 \%$ more likely to be allergically sensitized to one or more foods than those with lower levels of exposure, following adjustment for possible confounders including age, gender, presence of hay fever and asthma, and use of insecticides in the home. They did not have increased odds for sensitization to environmental allergens, however.

In addition, having one or more food allergies was more common if people had two dichlorophenol metabolites in their urine rather than one or none. Those with high urine levels (ie, 75th percentile and above) of two dichlorophenol metabolites had a significant $61 \%$ increased risk for having at least one food and one environmental allergy.

These findings concur with previous research suggesting that reduced microbial exposure can act as a trigger for atopy.

"Previous studies have shown that both food allergies and environmental pollution are increasing in the United States," said Jerschow. "The results of our study suggest these two trends might be linked, and that increased use of pesticides and other chemicals is associated with a higher prevalence of food allergies."

By Helen Albert, Senior medwireNews Reporter

\section{Reference}

Ann Allergy Asthma Immunol 2012; 109: 420-425 